\title{
Identification of Ligands and Translation to Clinical Applications
}

\author{
Uwe Haberkorn ${ }^{1-3}$, Walter Mier ${ }^{1,2}$, Klaus Kopka ${ }^{4}$, Christel Herold-Mende ${ }^{5}$, Annette Altmann ${ }^{1,2}$, and John Babich ${ }^{6}$ \\ ${ }^{1}$ Department of Nuclear Medicine, University Hospital Heidelberg, Heidelberg, Germany; ${ }^{2}$ Clinical Cooperation Unit Nuclear \\ Medicine, German Cancer Research Center (DKFZ), Heidelberg, Germany; ${ }^{3}$ Translational Lung Research Center Heidelberg \\ (TLRC), German Center for Lung Research (DZL), Heidelberg, Germany; ${ }^{4}$ Division of Radiopharmaceutical Chemistry, German \\ Cancer Research Center (DKFZ), Heidelberg, Germany; ${ }^{5}$ Division of Experimental Neurosurgery, Department of Neurosurgery, \\ University Hospital Heidelberg, Heidelberg, Germany; and ${ }^{6}$ Department of Radiopharmacy, Weill Cornell Medical College, \\ New York, New York
}

Technologic advances in molecular biology and biotechnology are increasingly being used for the development of new tumor-targeting tracers. In oncology, major progress has recently been achieved with peptidic and proteinaceous compounds. The development of new biocompatible molecules relies on the identification and validation of new target structures in close conjunction with the application of novel techniques. The identification of lead compounds by these techniques is followed by the screening of various derivatives of these molecules. Hence, high-throughput methods that generate vast libraries of epitopes have been applied. These libraries are screened to identify the few variants that bind with a high affinity to the target structure. A key feature of this strategy is the large number of candidate molecules that can be identified. Further evaluation and optimization of these molecules requires characterization of structure-function relationships and subsequent improvement with respect to binding, internalization, and biodistribution through a rational design of corresponding analogs.

Key Words: biotechnology; display methods; target validation; drug development

J Nucl Med 2017; 58:27S-33S

DOI: 10.2967/jnumed.116.186791

$\mathbf{M}$ diseases, surgery and external-beam radiation therapy-the standard therapeutic regimens - are intrinsically of limited scope, once tumor spread has occurred. In contrast, molecularly targeted radiotherapeutics have the potential to achieve cure, under the assumptions of ideal conditions. The implementation of novel molecular entities in clinical practice requires a line of research and development that relates the results of basic science to knowledge of pharmacology, radiochemistry and, finally, medical needs. Researchers may, of course, learn from the experiences of pharmaceutical companies but should be aware that there are important design and development differences between pharmaceuticals and radiophar-

Received Feb. 17, 2017; revision accepted Mar. 15, 2017.

For correspondence or reprints contact: Uwe Haberkorn, Department of Nuclear Medicine, University Hospital Heidelberg, Im Neuenheimer Feld 400, 69120 Heidelberg, Germany.

E-mail: uwe.haberkorn@med.uni-heidelberg.de

COPYRIGHT (C 2017 by the Society of Nuclear Medicine and Molecular Imaging. maceuticals. The target for a radiopharmaceutical may not necessarily be causally involved in the development of disease; it may just be associated with the disease phenotype. Proteins controlling cellular homeostasis may not be the best choice as a target because the difference between the normal state and the diseased state may be only modest. Also, there is no need to restore equilibrium or the genetic repertoire of cancer cells. Basically, the key goal is to direct as much radioactivity as possible to the tumor for an extended period of time to allow the deposition of sufficient energy to kill cancer cells but not normal tissues. Therefore, carrier molecules that specifically bind to and transport radionuclides inside tumor cells and have minimal or reduced affinity for or interaction with normal tissue are required.

In recent years, researchers also have learned that cancer cells may not be the only cells that represent or possess potential targets within the tumor mass; cellular components of the tumor stroma and microenvironment may also serve as targets. Therefore, nontumor cells and matricellular proteins can represent potential structures for ligand development. The process of tracer development can be described as a chain of consecutive steps starting with defining the clinical need. The next steps are target identification, target validation, selection or production of the appropriate tools or target structures, identification of possible structural leads, ligand synthesis, in vitro screening and subsequent radiolabeling of ligands identified by the screening, and iterative evaluation of in vitro and in vivo biologic characteristics and behaviors. The outcome of the in vitro and in vivo validation commonly results in the disqualification of many ligands or the realization of the need to modulate the structure to enhance affinity or stability. Finally, an initial proof-of-concept study may be done in patients (Fig. 1).

\section{TARGET IDENTIFICATION AND TARGET VALIDATION}

Usually target identification is performed by screening literature reports based on autopsy studies, genetic linkage studies, genomewide association studies, RNA interference studies, proteomics, forward and reverse genetics, and large-scale datasets from genomics $(1,2)$. However, there are concerns about the reproducibility of the published data because analyses by pharmaceutical companies, such as Bayer and Amgen, and academia did not show consistency between findings reported even in very highly ranked journals and in-house data (2-5). The analysis conducted by Amgen revealed that in only 6 of 57 projects could the findings reported in the literature be confirmed. The Bayer analysis led to similar findings, with 14 of 67 projects being confirmed. These data may explain why the rates of success of phase 2 studies dropped 


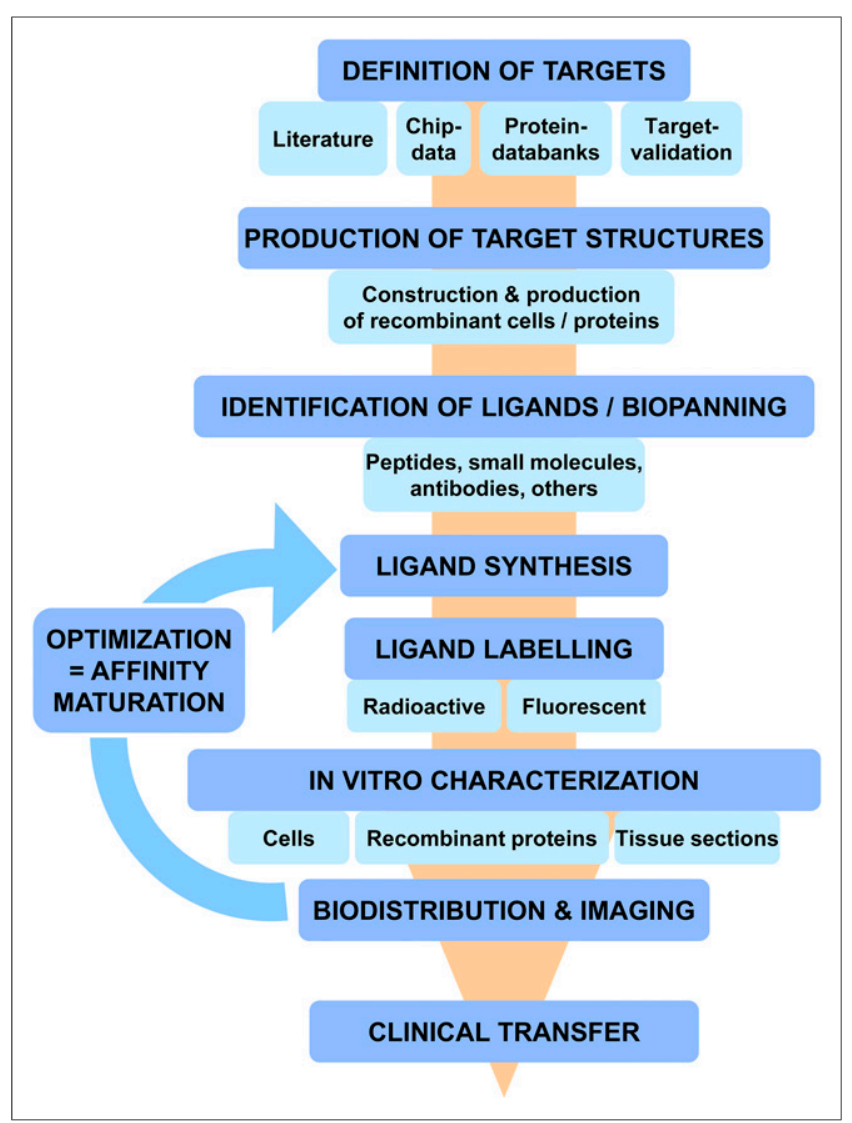

FIGURE 1. Process of tracer development from target identification to proof of concept in patients.

from $28 \%$ to $18 \%$ in recent years (6). Therefore, uncertainty about even well-known and established targets remains unless there has been a proof-of-concept study in patients. Good examples are somatostatin receptor ligands and ligands against prostate-specific membrane antigen (PSMA); for these ligands, peptides and smallmolecule inhibitors were developed by the pharmaceutical industry and applied in patients before being used as lead structures for tracer development $(7,8)$. For new and less characterized targets, there may be no other choice than repetition to confirm the data. Since analyses in yeast and mammalian cells have shown that messenger RNA (mRNA) levels are not reliable predictors of corresponding protein levels (9), gene expression data are insufficient and the need for verification at the protein level remains.

Critical issues in the development of radiopharmaceuticals involve either the target or the ligand. Regarding the target, researchers need to determine what the medical problem is; whether there is a biologic or biochemical hypothesis; whether there is a known target; whether upregulation of the target occurs in tumor or in normal tissue; whether the target is localized in membranes or within cells; whether the extracellular domain is large enough; whether the target degrades with shedding and, if so, what is left at the plasma membrane; and whether internalization or recycling occurs.

The first step is to determine whether it makes sense to engage in tracer development for a particular target. This step leads to the identification of a medical need and the corresponding biologic or biochemical hypothesis that can be related to a target. Several essential features are needed for triggering the efficient uptake of radiotracers.

\section{Upregulation in Tumor Tissue}

Once the target is identified, expression levels in tumor and normal tissue must be defined. If the target is expressed predominantly in the tumor, heterogeneity may be an issue. Intratumoral heterogeneity may cause treatment failure because of the presence of divergent phenotypes with different susceptibilities to a particular therapeutic intervention. In addition, intratumoral heterogeneity may lead to sampling bias when biopsies are taken (10). These effects may contribute to the failure observed for RNA expression or DNA copy number analysis with respect to the prediction of a therapy response $(11,12)$, but it also may be responsible for the failure of endoradiotherapy (i.e., target-negative cells may be responsible for tumor regrowth).

\section{Extracellular Localization}

The limited success of ${ }^{111}$ In-capromab pendetide (ProstaScint; Cytogen Corp.) in the detection of PSMA-positive prostate cancer originates from the fact that the antibody used binds to the intracellular domain of the target antigen. This fact demonstrates the importance of gaining knowledge about the localization of the target (membrane-associated vs. intracellular) and the accessibility of the targeting molecule. In addition, an extracellular domain of sufficient size should be available, at least when small molecules or peptides are used. Intracellular proteins or membrane proteins with intracellularly localized targets, such as tyrosine kinases, are generally suboptimal. Exceptions are thymidine kinase 1 and hexokinase, whose radiolabeled substrates-3'-deoxy3 '-fluorothymidine and FDG-are metabolically trapped inside the cell on uptake via transport molecules. Another intracellular target with potential is melanin, which has been exploited as a target for the endoradiotherapeutic treatment of metastatic melanoma (13). For larger molecules, such as antibodies, the plasma membrane certainly is a considerable obstacle.

\section{No Shedding on Ligand Binding}

Another factor that may be easily overlooked is the possibility of degradation of the target with shedding. Many antibodies with a high affinity have been developed against mucin 1, but they recognize epitopes within the highly immunogenic $\alpha$-chain tandemrepeat array. However, the mucin $1 \alpha$-chain is shed into the peripheral circulation, where it can form complexes with circulating antibodies-thereby limiting their ability to reach mucin 1 -overexpressing tumor cells-as well as redirect radioactivity to other, normal organs, such as the liver and reticuloendothelial system $(14,15)$. Thus, the critical questions are: What is left at the plasma membrane? Can it be targeted effectively?

\section{Internalization After Ligand Binding}

The final property of antigens primarily applied to therapeutic applications relates to their trafficking. Internalization followed by recycling of the target is a favorable property of the target because it leads to the transport of radioactivity into tumor cells, which can be used for both diagnosis and endoradiotherapy. Recycling of the protein allows the target to reappear at the membrane surface, enabling subsequent ligand interactions.

\section{Presence of Family Members or Splice Variants with High Homology at Protein Level}

Expression on other family members or splice variants in nontarget tissues may result in a high background. In these situations, strategies for avoiding binding to regions with common amino acid sequences are needed. 


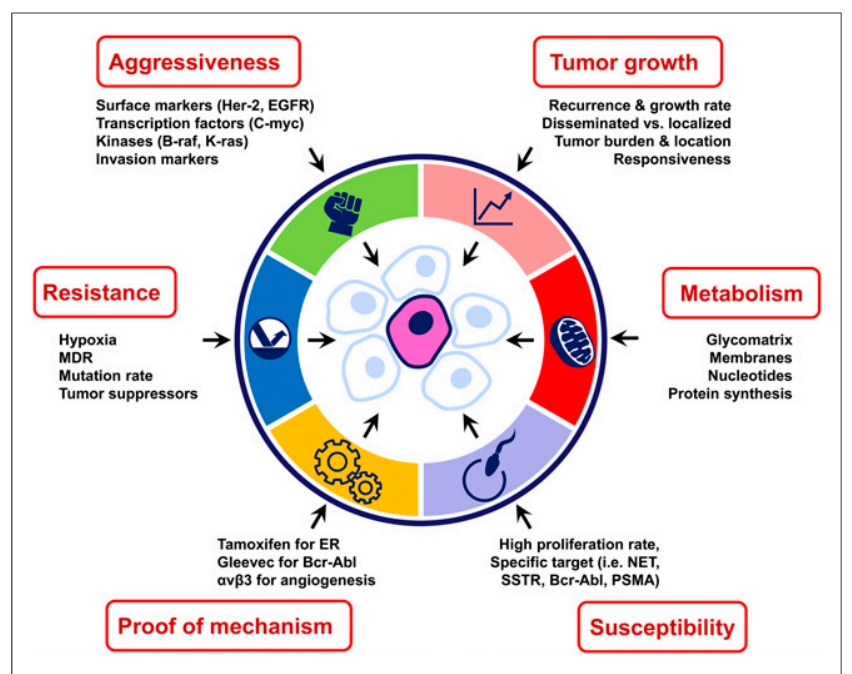

FIGURE 2. Tumor characteristics as basis for selection of targets for directed therapies of cancers. EGFR = epidermal growth factor receptor; ER $=$ estrogen receptor; Gleevec $=$ imatinib mesylate (Novartis); Her-2 = human epidermal growth factor 2; MDR = multidrug resistance; $\mathrm{NET}=$ neuroendocrine tumor; SSTR $=$ somatostatin receptor.

\section{IDENTIFICATION OF LIGANDS}

Regarding the ligand, researchers need to determine whether it functions as an enzyme, receptor, or something else; whether it has a known lead structure; whether it is an antibody, small molecule, or peptide; whether it is an inhibitor or a substrate; its affinity and stability; how many receptor molecules are at the plasma membrane; and its pharmacokinetics, such as whether it binds to plasma proteins and whether its excretion pathway is renal or intestinal.

The presence of a target with a known function (such as an enzyme or a receptor related to the biologic behavior of a tumor) (Fig. 2) or a known lead structure (likely a natural substrate or ligand) that defines binding to or interaction with the target is fortuitous. Somatostatin receptor imaging and therapy can be considered the paradigm for receptor targeting using peptides. A high affinity of a peptide for its receptor, accompanied by internalization of the receptor-peptide complex, facilitates retention of the radionuclide in receptor-expressing tumors, whereas the relatively small size of a peptide facilitates rapid clearance from the blood.

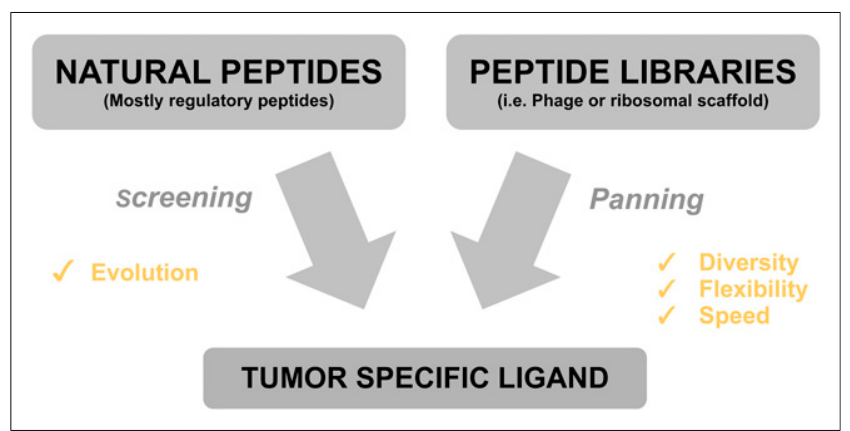

FIGURE 3. Two alternative sources for potential peptide ligands: use of known tumor-binding peptides and de novo identification of peptides from random libraries. On radiolabeling, specific ligand is used as mimotope binding target of interest.
For PSMA ligands, imaging and endoradiotherapy are done either with a PSMA-binding antibody or with a radiolabeled small molecule that is recognized by the enzymatic domain of PSMA (7). These two examples can be seen as prime examples for rational design. In rational design, information obtained from the study of structure-activity relationships and the conformational properties of peptide structures is used. This approach relies on the identification of lead compounds followed by screening of numerous derivative compounds.

In contrast to rational design, combinatorial chemistry involves the synthesis of a vast library of possible variants of the molecule of interest and screening of the whole population to identify the few variants that show the desired interaction (Fig. 3). The attractive feature of this concept is the generation of a large number of molecules that can be used for further identification and evaluation of binders. For example, the diversity of a hexapeptide library randomizing the 20 natural amino acids at each position amounts to a sequence space of 64 million variants. The critical issue in this process is the development of sensitive and efficient high-throughput screening techniques that allow the rapid identification of molecules with the desired properties. This step is followed by characterization of the structure-activity relationships for the identified compounds. Subsequently, further improvements can be obtained by mutation analysis or rational design of analogs (16).

Molecular libraries that provide a high degree of structural diversity may be created by means of chemical or biologic libraries. Chemical libraries consist of compounds produced by random or directed synthesis and generally displayed on solid supports such as activated beads, pins, or batch arrays. Biologic libraries are developed by use of genetically encoded and expression systems and usually consist of RNA, DNA, proteins, and peptides (17). The recombinant techniques applied are random mutation, DNA shuffling, and display techniques such as phagedisplay, ribosome display, or mRNA display. After the identification of lead structures, further improvements can be obtained by mutation and amplification to create a new pool of diverse molecules for further screening rounds. This iterative process of mutation, selection, and amplification to evolve the best-fit molecule is known as "directed evolution" (16).

\section{BIOTECHNOLOGY METHODS FOR IDENTIFICATION OF NEW LIGANDS: DISPLAY SYSTEMS}

Display systems are applied for the selection of molecules from libraries in which peptides or proteins such as antibodies (phenotype) are physically linked to their corresponding encoding sequences (genotype). In addition, these systems can be used to modify the biophysical properties of the displayed molecules by evolution through cycles of mutation, selection, and replication. In principle, two types of display systems are available: cell-based systems, such as phage display (Fig. 4) or cell surface display, and cell-free systems, such as ribosome display and mRNA display.

In phage display libraries, peptide, antibody, or other protein libraries are fused to the carboxy-terminal domain of minor coat protein III or VII on the surface of a filamentous phage (16). The ligand binding to the target is detected using the recombinant receptor molecules or cell lines. Subsequently, the binding phages are amplified by infection of bacteria. This approach allows the selection of clones from large pools of sequences $\left(>10^{10}\right.$ clones) within weeks. Selection is followed by determination of the amino 


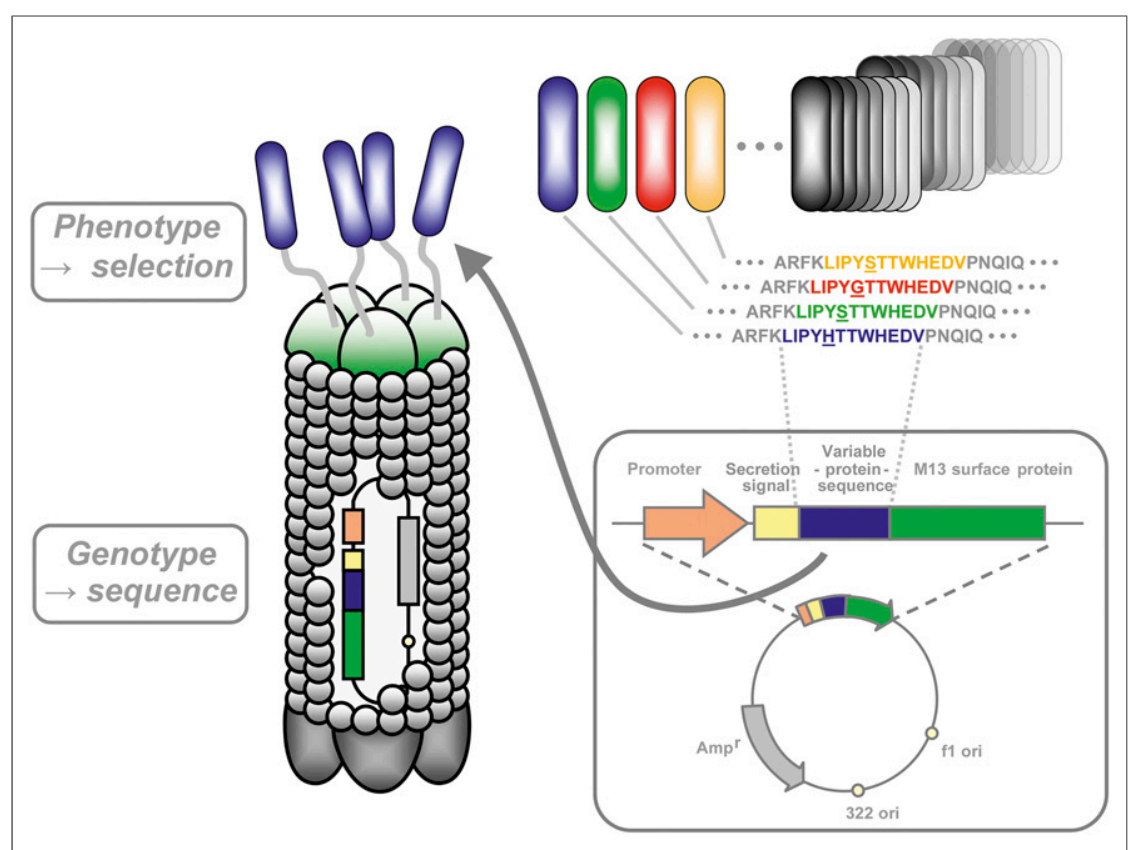

FIGURE 4. Composition of peptide libraries presented by phage templates. Bacteriophages constitute prototype of display system in which peptide or protein motif is linked to genetic information that encodes its sequence. Insertion of random sequences into plasmids of bacteriophages allows production of random libraries containing diversity required to rapidly screen billions of different peptide sequences. Panning process is applied to select motifs that specifically bind tumor-specific targets.

acid sequence displayed on a phage by sequencing of the section of the phage genome in which the library is fused to the coat protein. Selection can be done by exposure of the library to recombinant target proteins or cells or even in animals. This approach has been used in various applications, such as in the mapping and mimicking of epitopes, the identification of new receptors and natural ligands, high-affinity antibodies and analogs, the isolation of specific antigens binding to bioactive compounds, the production of novel enzyme inhibitors and DNA-binding proteins, and the probing of cellular and tissue-specific processes. Phage display has been successfully applied for the identification of peptides with a high specificity for target tumor neovasculature or a variety of human tumor cells $(16,18-33)$.

In vitro selection systems such as ribosome display and mRNA display represent an alternative to phage display. They are technically demanding but have two advantages: the ability to handle very large libraries $\left(10^{12}-10^{13}\right.$ different sequences or potential molecules) and the possibility of using polymerase chain reaction (PCR) amplification steps to introduce further diversity into the system. Such diversity may be used for the evolution of proteins through iteration of random mutagenesis and selection, a process known as affinity maturation $(16,34,35)$. Both ribosome display and mRNA display have been used to select linear peptides or single-chain antibodies that bind to protein targets with low picomolar affinities.

Ribosome display is based on the translation of libraries consisting of mRNA molecules with stoichiometric quantities of ribosomes. Because the mRNA molecules in the library have no stop codon, the scanning process for the ribosomes can be extended to the ends of the mRNA molecules. The corresponding polypeptide emerges from the ribosomes, and the lack of a stop codon ensures that its end is fixed within the ribosomal tunnel and its last amino acid is connected to the peptidyl-transfer RNA. The absence of a stop codon prevents the binding of release factors, which normally catalyze the release of the polypeptide from the ribosomes. This process leads to the formation of a protein-ribosome-RNA complex, which connects the phenotype (peptides, antibodies, or miniproteins) to the genotype (RNA) (36-39). The steps of ribosome display are construction of a large DNA library encoding peptides, antibodies, or miniproteins fused in frame to a C-terminal spacer; in vitro transcription of the library into mRNA; and in vitro translation. Thereafter, proteinmRNA-ribosome complexes are exposed to the target structure. Washing to remove nonbinding molecules leads to enrichment of binding molecules. The binding molecules are amplified by reverse transcription-PCR of the mRNA; this step is followed by the next round of transcription, translation, and exposure. During the amplification process, diversity may be further increased with errorprone PCR. High-affinity binding molecules are usually obtained after 3-6 panning rounds $(35,38,39)$.

Ribosome display libraries have been used for the selection of peptides binding to several targets, such as prostate-specific antigen, $\delta$-like ligand 4 , streptavidin, and lysozyme, or to identify the main antigenic polypeptides of Staphylococcus aureus. The affinity of these peptides ranged from 7.2 to $140 \mathrm{nM}$. Similar results have been obtained for single-chain variable fragments ( $\mathrm{scFv}$ ) using targets such as the GCN4 leucine zipper, progesterone, and fluorescein. All selected $\mathrm{scFv}$ acquired genetic mutations during the biopanning process. A comparison of the isolated $\mathrm{scFv}$ and their progenitors revealed that the selected $\mathrm{scFv}$ had mutations due to errors introduced by the activity of DNA polymerase used for the amplification step and that these mutations led to up to 40-fold improvements in their affinities for the antigen. The best scFv had affinities in the low picomolar range and could be further improved by off-rate selection and error-prone PCR (36).

The strategy for mRNA display is similar to that for ribosome display: a complex between mRNA and the polypeptide encoded by the mRNA can be applied for the selection process (37). The method differs from ribosome display in the covalent nature of the linkage between the mRNA and the protein in the mRNA-protein complex, achieved by linking the two molecules through a small adapter molecule, typically puromycin. The steps include transcription of a large DNA library encoding the molecules of interest, which are free of stop codons, into mRNA; ligation of an adapter molecule to the $3^{\prime}$ ends; and in vitro translation $(16,37)$. This process results in a peptide bond between the adapter molecule and the C-terminal amino acid residue in the polypeptide chain. After the introduction of complementary DNA (cDNA) chains by reverse transcription for stabilization and easy recovery of the genetic information, the complementary DNA-mRNA protein library is exposed to the target. Binders are isolated by affinity chromatography or immunoprecipitation of the target structure. Amplification for the 
next panning cycle is then realized using the complementary DNA. In general, 4-10 biopanning rounds may be necessary to select proteins with a nanomolar affinity for a given target. This process has been used for engineered libraries of linear peptides, structurally constrained peptides, variable heavy domains of antibodies, and single-chain antibodies. When targets such as an antic-Myc antibody or streptavidin were used, binders with a binding constant as low as $2.5 \mathrm{nM}$ were found (40).

\section{AFFINITY AND STABILITY OR SCYLLA AND CHARYBDIS: IS THERE A SOLUTION?}

A general problem for linear peptides is their degradation by peptidases. This degradation inevitably leads to a stability problem, with a plasma half-life in the range of several minutes (26-30). Stability may be increased by shortening the peptide after identification of the essential binding sequence using an alanine scan, exchange of single amino acids, introduction of D-amino acids, peptide cyclization, and coupling to chelators such as DOTA. Although this approach may lead to increased metabolic stability, these changes frequently cause a decrease in affinity $(29,30,41)$. Therefore, the strategy of first identifying a linear peptide with acceptable affinity and then trying to make it stable while retaining its affinity is a circular approach that will keep a scientist busy for years.

An instructive case is that of peptide FROP-1 (FROP), which was identified by biopanning with a linear peptide library against thyroid carcinoma cells (30). The original peptide showed binding to follicular thyroid carcinoma as well as anaplastic thyroid carcinoma, mammary carcinoma, cervix carcinoma, and prostate carcinoma and a low affinity for human umbilical vein endothelial cells or immortalized keratinocytes. In MCF-7 cells, $78 \%$ of the bound activity was internalized after $10 \mathrm{~min}$ of incubation. However, stability experiments in human serum revealed a degradation product after 15 min. Further analysis indicated that degradation started with the cleavage of the N-terminal amino acid (glutamate). Tumor uptake of the ${ }^{125}$ I-labeled peptide increased for $45 \mathrm{~min}$ in nude mouse models, reaching 3.6 percentage injected dose per gram $(\% \mathrm{ID} / \mathrm{g})$ for FRO82-2 tumors and $3.8 \% \mathrm{ID} / \mathrm{g}$ for MCF-7 tumors. To improve stability, DOTA was coupled to the peptide (42). Despite its excellent tumor-binding capacity, FROP-DOTA had slow binding kinetics. Biodistribution studies showed that the clearance was very fast and, together with the slow binding kinetics observed in vitro, prevented significant tumor accumulation (Fig. 5). The next step investigated whether the conjugation of polyethylene glycol to FROPDOTA would result in a derivative with a prolonged residence time in the blood (43). The binding kinetics of pegylated FROP-DOTA

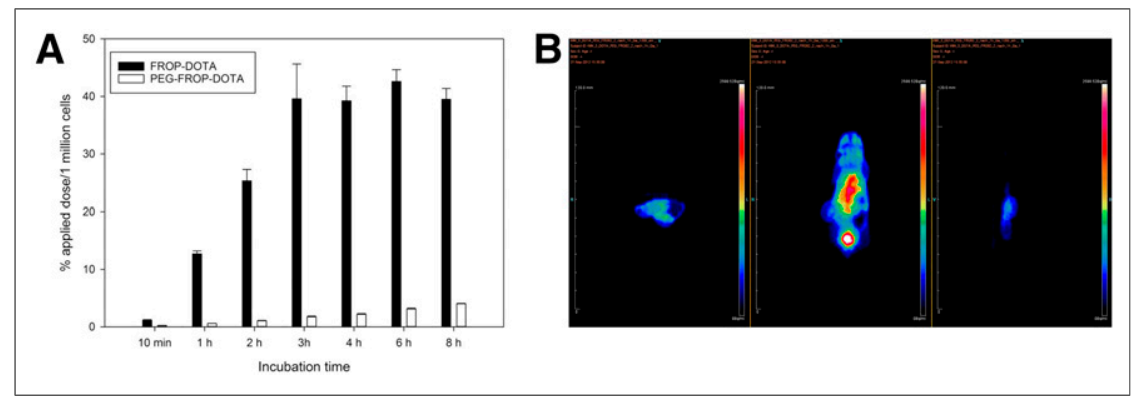

FIGURE 5. (A) Kinetics of binding of FROP-DOTA and pegylated FROP-DOTA (PEG-FROPDOTA) in FRO82-2 cells. (B) Small-animal PET of FRO82-2 tumor-bearing mouse. were even slower than those of FROP-DOTA. Biodistribution studies of the labeled conjugate in mice bearing human FRO82-2 tumors revealed a time-dependent increase in uptake of the pegylated peptide (2.3\% ID/g at $2 \mathrm{~h}$ after tracer administration) (43). However, as shown in Figure 5B, the tumor was barely seen. Therefore, the project was not continued.

Biomolecules derived from constrained peptides have advantages over linear peptides because they are more similar in structure to globular proteins as a result of their smaller loss of entropy on target binding $(16,44)$. Binding surfaces for proteins have been engineered on the faces of helical bundles (a small protein fold composed of several $\alpha$-helices) or similar scaffolds $(34,44)$. Some scaffolds, such as those based on lipocalins, the 10th fibronectin type III domain, or helical bundles such as Affibody molecules (which are derivatives of the $\mathrm{Z}$ domain of $S$. aureus protein A), have been successfully used for the selection of high-affinity binders (45). The fibronectin type III domain, which has an immunoglobulinlike fold but is smaller than a heavy chain variable domain, was used to generate mRNA display libraries in which the diversity was concentrated in 3 exposed loops by randomizing 21 residues in these loops (analogously to antibody complementarity-determining regions). Selection against tumor necrosis factor- $\alpha$ and affinity maturation of the identified ligand with error-prone PCR revealed Fn3-like domains with affinities of about 20 pM (46). Similar results were obtained with vascular endothelium growth factor receptor 2. Furthermore, a library based on the knottin trypsin inhibitor EETI-II was designed by randomization of the 6 residues of the trypsin-binding moiety. mRNA display revealed trypsinbinding peptides with a high homology to wild-type EETI-II (44).

When libraries based on the trypsin inhibitors sunflower trypsin inhibitor and Min23 were applied, peptides were identified by phage display as well as ribosome display for Dll4 and $\alpha_{\mathrm{v}} \beta_{6}$-integrin (3133). The Dll4 peptides showed a high affinity, in the range of $4-40 \mathrm{nM}$, and a serum stability of up to $10 \mathrm{~d}$. When a membrane proteome of HNO97 tumor cells (head and neck squamous cell carcinoma) fractionated by the ProteomeLab PF2D system (Beckman Coulter) and corresponding HNO97 cells for phage display with a sunflower trypsin inhibitor library were used, a novel $\alpha_{\mathrm{v}} \beta_{6}$-integrin-binding peptide (SFITGv6) with stability over a period of $24 \mathrm{~h}$, a high affinity (diffusion constant, $14.8 \mathrm{nM}$ ) for $\alpha_{\mathrm{v}} \beta_{6}$-integrin, and an internalization ratio of $37.5 \%$ was identified (33). Small-animal PET imaging and biodistribution studies of mice with HNO97 xenografts showed tumor-specific accumulation of ${ }^{68} \mathrm{Ga}$ - and ${ }^{177}$ Lu-labeled DOTA-SFITGv6. Peptide histochemistry revealed strong and homogeneous binding of biotin-labeled SFITGv6 to head and neck squamous cell carcinoma and breast cancer- and lung cancer-derived brain metastases. Finally, initial PET/CT scans of head and neck squamous cell carcinoma and non-small cell lung cancer patients showed SFITGv6 accumulation specifically in tumors and not in inflammatory lesions (Fig. 6).

Affibody molecules, 3-helix-bundle proteins with 58 amino acids derived from the $\mathrm{Z}$ domain of $S$. aureus protein A, may also be useful as scaffolds. A phage display library was generated by random mutagenesis of residues from the $\mathrm{Z}$ domain-binding surface, and binders were isolated for some targets, including human insulin, HIV type 1 gp120, human apolipoprotein A-I, and 


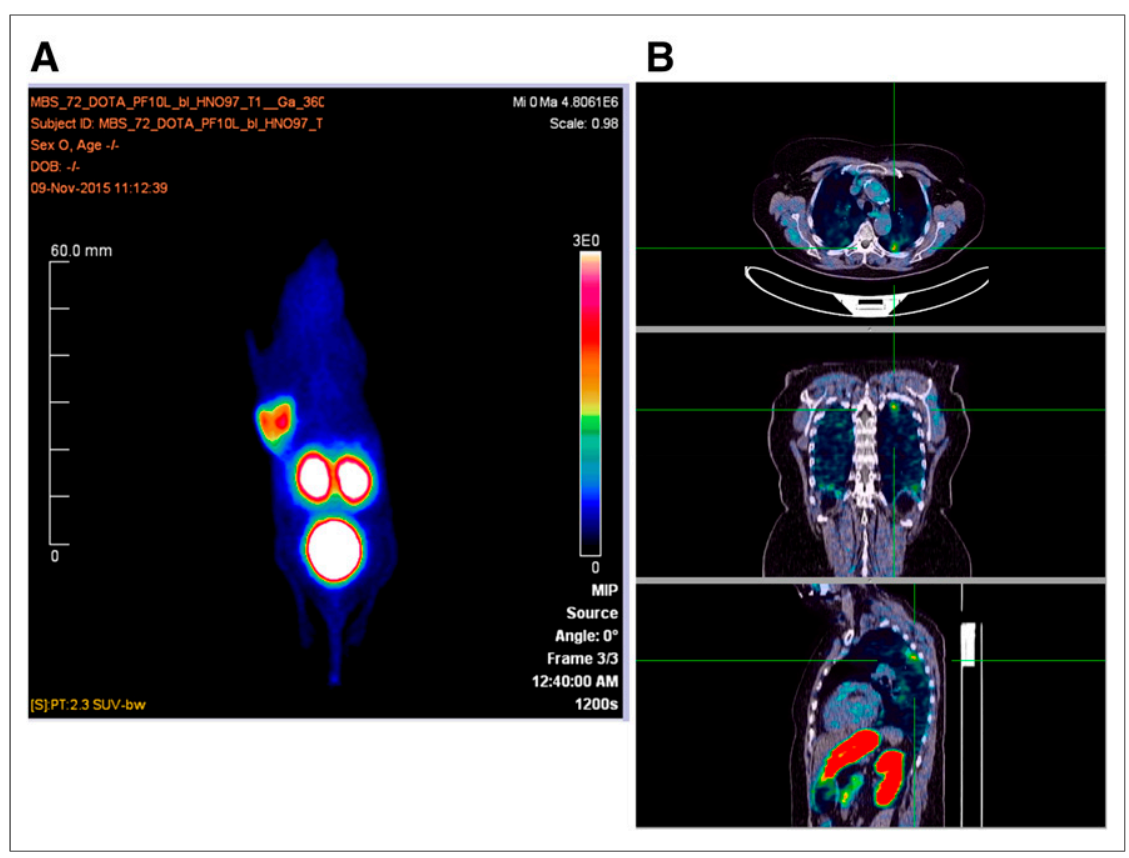

FIGURE 6. (A) Small-animal PET showing tumor, kidneys, and bladder after administration of ${ }^{68} \mathrm{Ga}$-labeled SFITGv6 in tumor-bearing mouse. (B) Transaxial, coronal, and sagittal slices from patient with lung cancer. Tumor can be seen in left upper lung.

human epidermal growth factor 2 (HER2) (44,47). Initial studies with an anti-HER2 Affibody (ZHER2:342) revealed selective uptake in HER2-expressing tumors, with excellent image contrast. However, the small size $(7 \mathrm{kDa})$ of the molecule caused rapid glomerular filtration and high kidney uptake. Therefore, a dimeric Affibody was fused to albumin, and this construct reduced renal uptake by a factor of 25. Treatment of a tumor that had high HER2 expression (SKOV-3) with 17 or $22 \mathrm{MBq}$ of a ${ }^{177} \mathrm{Lu}$-labeled compound $\left[{ }^{177} \mathrm{Lu}-\right.$ CHX-A"'-DTPA-ABD-(ZHER2:342) 2 ] completely prevented the growth of the tumor. Even in xenografts with low HER2 expression (LS174T), therapy with this molecule caused a small but significant increase in survival (48).

\section{RATIONAL DESIGN: TRANSFORMATION INTO RADIOTRACERS}

For clinical applications, the identification of novel binders must be pursued through appropriate modifications. The aim of such modifications is multifold. First, the targeting molecule must be linked to a radionuclide of interest. Although proteins can be readily labeled with radionuclides of iodine for preliminary examinations, small molecules require sophisticated methods for the incorporation of a radionuclide such as ${ }^{11} \mathrm{C}$ or ${ }^{18} \mathrm{~F}$. These isotopes can be applied without extensive modification of the properties of the molecule to be labeled. Once the applicability of the potential radioligand has been proven, routine labeling strategies must be established. In this respect, folded peptides have strong advantages over linear peptides because their linkage to complex chelating agents will not have destructive effects on stable molecules. As mentioned earlier, somatostatin receptorbinding molecules were the prototypes of peptide radiopharmaceuticals. Their development took advantage of the efforts of Sandoz in a large program started to develop peptide hormone therapeutics. At present, drug development is confronted with the broad availability of large molecules that do not fulfill the classic rules of drug development. Future efforts must be focused on strategies that will allow researchers to close the gap between drugs with a molecular mass of less than $500 \mathrm{Da}$-corresponding to the traditional understanding of what constitutes a drug - and newer, proteinaceous drug candidates.

Finally, researchers developing radiotracers must consider the fact that current, highly efficient biotechnology techniques increasingly are leading to the identification of target molecules with a low abundance. As a consequence, strategies that enable high drug load ratios, such as conjugation of multivalent chelator complexes, must be developed (49).

\section{CONCLUSION}

Advances in understanding of the biology of tumors stimulates the development of new molecules for the targeted therapy of cancer. The potential targets can be cloned and thereby made available as potential diagnostic or drug discovery targets for further structural exploration. Generally, there are two sources of potential peptide ligands - the most straightforward being peptides previously known to bind to receptors expressed on tumor cells. However, such peptides have been the focus of research for many years, and the chances of identifying a stellar binder seem to be fading. The second option-the de novo identification of peptides from libraries-provides access to peptide sequences beyond the overworked pool of known tumor-binding peptides. In this process, target selection and validation are the most critical components. Biotechnology methods offer researchers a large number and variety of sequences or chemical structures to be considered as biomolecules applicable for diagnosis and treatment. Furthermore, novel scaffold structures that may improve the affinity and stability of binding molecules can be applied. Tracers based on these new molecular entities represent promising opportunities for the establishment of new diagnostic and therapeutic procedures.

\section{DISCLOSURE}

No potential conflict of interest relevant to this article was reported.

\section{REFERENCES}

1. Lindsay MA. Target discovery. Nat Rev Drug Discov. 2003;2:831-838.

2. Stock JK, Jones NP, Hammonds T, Roffey J, Dillon C. Addressing the right targets in oncology: challenges and alternative approaches. J Biomol Screen. 2015;20:305-317.

3. Prinz F, Schlange T, Asadullah K. Believe it or not: how much can we rely on published data on potential drug targets? Nat Rev Drug Discov. 2011;10:712.

4. Begley CG, Ellis LM. Drug development: raise standards for preclinical cancer research. Nature. 2012;483:531-533.

5. Blagg J, Workman P. Chemical biology approaches to target validation in cancer. Curr Opin Pharmacol. 2014;17:87-100.

6. Arrowsmith J. Phase II failures: 2008-2010. Nat Rev Drug Discov. 2011;10:328-329.

7. Haberkorn U, Eder M, Kopka K, Babich JW, Eisenhut M. New strategies in prostate cancer: prostate-specific membrane antigen (PSMA) ligands for diagnosis and therapy. Clin Cancer Res. 2016;22:9-15. 
8. Afshar-Oromieh A, Babich JW, Kratochwil C, et al. The rise of PSMA ligands for diagnosis and therapy of prostate cancer. J Nucl Med. 2016;57(suppl 3):79S89 S.

9. Pradet-Balade B, Boulmé F, Beug H, Müllner EW, Garcia-Sanz JA. Translation control: bridging the gap between genomics and proteomics? Trends Biochem Sci. 2001;26:225-229.

10. Yap TA, Gerlinger M, Futreal PA, Pusztai L, Swanton C. Intratumoral heterogeneity: seeing the wood for the trees. Sci Transl Med. 2012;4:127ps10.

11. Poste G. Bring on the biomarkers. Nature. 2011;469:156-157.

12. Sotiriou C, Pusztai L. Gene-expression signatures in breast cancer. $N$ Engl $J$ Med. 2009;360:790-800.

13. Mier W, Kratochwil C, Hassel JC, et al. Radiopharmaceutical therapy of patients with metastasized melanoma with the melanin-binding benzamide ${ }^{131} \mathrm{I}-\mathrm{BA} 52 . J$ Nucl Med. 2014;55:9-14.

14. Levitin F, Stern O, Weiss M, et al. The MUC1 SEA module is a self-cleaving domain. J Biol Chem. 2005;280:33374-33386.

15. Rubinstein DB, Karmely M, Ziv R, et al. MUC1/X protein immunization enhances cDNA immunization in generating anti-MUC1 alpha/beta junction antibodies that target malignant cells. Cancer Res. 2006;66:11247-11253.

16. Haberkorn U, Eisenhut M, Altmann A, Mier W. Endoradiotherapy with peptides: status and future development. Curr Med Chem. 2008;15:219-234.

17. Wong D, Robertson R. Applying combinatorial chemistry and biology to food research. J Agric Food Chem. 2004;52:7187-7198.

18. Braun GB, Sugahara KN, Yu OM, et al. Urokinase-controlled tumor penetrating peptide. J Control Release. 2016;232:188-195.

19. Laakkonen P, Zhang L, Ruoslahti E. Peptide targeting of tumor lymph vessels. Ann N Y Acad Sci. 2008;1131:37-43.

20. Lee SM, Lee EJ, Hong HY, et al. Targeting bladder tumor cells in vivo and in the urine with a peptide identified by phage display. Mol Cancer Res. 2007;5: 11-19.

21. Ruoslahti E. Targeting tumor vasculature with homing peptides from phage display. Semin Cancer Biol. 2000;10:435-442.

22. Mandelin J, Cardó-Vila M, Driessen WH, et al. Selection and identification of ligand peptides targeting a model of castrate-resistant osteogenic prostate cancer and their receptors. Proc Natl Acad Sci USA. 2015;112:3776-3781.

23. Vidal CI, Mintz PJ, Lu K, et al. An HSP90-mimic peptide revealed by fingerprinting the pool of antibodies from ovarian cancer patients. Oncogene. 2004;23:8859-8867.

24. Larimer BM, Thomas WD, Smith GP, Deutscher SL. Affinity maturation of an ERBB2-targeted SPECT imaging peptide by in vivo phage display. Mol Imaging Biol. 2014;16:449-458.

25. Deutscher SL, Figueroa SD, Kumar SR. Tumor targeting and SPECT imaging properties of an ${ }^{111} \mathrm{In}$-labeled galectin-3 binding peptide in prostate carcinoma. Nucl Med Biol. 2009;36:137-146.

26. Zitzmann S, Mier W, Schad A, et al. A new prostate carcinoma binding peptide (DUP-1) for tumor imaging and therapy. Clin Cancer Res. 2005;11:139-146.

27. Askoxylakis V, Zitzmann S, Mier W, et al. Preclinical evaluation of the breast cancer cell binding peptide p160. Clin Cancer Res. 2005;11:6705-6712.

28. Askoxylakis V, Garcia-Boy R, Rana S, et al. A new peptide ligand for targeting human carbonic anhydrase IX, identified through the phage display technology. PLoS One. 2010;5:e15962.
29. Nothelfer EM, Zitzmann-Kolbe S, Garcia-Boy R, et al. Identification and characterization of a peptide with affinity to head and neck cancer. $\mathrm{J} \mathrm{Nucl} \mathrm{Med}$. 2009;50:426-434.

30. Zitzmann S, Kramer S, Mier W, et al. Identification and evaluation of a new tumor cell-binding peptide, FROP-1. J Nucl Med. 2007;48:965-972.

31. Zoller F, Markert A, Barthe P, et al. A disulfide-constrained miniprotein with striking tumor-binding specificity developed by ribosome display. Angew Chem Int Ed Engl. 2013;52:11760-11764.

32. Zoller F, Markert A, Barthe P, et al. Combination of phage display and molecular grafting generates highly specific tumor-targeting miniproteins. Angew Chem Int Ed Engl. 2012;51:13136-13139.

33. Altmann A, Sauter M, Roesch S, et al. Identification of a novel ITG $\alpha_{\mathrm{v}} \beta_{6}$-binding peptide using protein separation and phage display. Clin Cancer Res. In press.

34. Lipovsek D, Plueckthun A. In-vitro protein evolution by ribosome display and mRNA display. J Immunol Methods. 2004;290:51-67.

35. Marr A, Markert A, Altmann A, Askoxylakis V, Haberkorn U. Biotechnology techniques for the development of new tumor specific peptides. Methods. 2011;55: 215-222.

36. Hanes J, Schaffitzel C, Knappik A, Plückthun A. Picomolar affinity antibodies from a fully synthetic naive library selected and evolved by ribosome display. Nat Biotechnol. 2000;18:1287-1292.

37. Roberts RW, Szostak JW. RNA-peptide fusions for the in vitro selection of peptides and proteins. Proc Natl Acad Sci USA. 1997;94:12297-12302.

38. Zahnd C, Amstutz P, Plückthun A. Ribosome display: selecting and evolving proteins in vitro that specifically bind to a target. Nat Methods. 2007;4:269-279.

39. Amstutz P, Forrer P, Zahnd C, Plückthun A. In vitro display technologies: novel developments and applications. Curr Opin Biotechnol. 2001;12:400-405.

40. Wilson DS, Keefe AD, Szostak JW. The use of mRNA display to select highaffinity protein-binding peptides. Proc Natl Acad Sci USA. 2001;98:3750-3755.

41. Askoxylakis V, Zitzmann-Kolbe S, Zoller F, et al. Challenges in optimizing a prostate carcinoma binding peptide, identified through the phage display technology. Molecules. 2011;16:1559-1578.

42. Mier W, Zitzmann S, Krämer S, et al. Influence of chelate conjugation on a newly identified tumor-targeting peptide. J Nucl Med. 2007;48:1545-1552.

43. Mier W, Krämer S, Zitzmann S, et al. PEGylation enables the specific tumor accumulation of a peptide identified by phage display. Org Biomol Chem. 2013;11:2706-2711.

44. Nygren PA, Uhlen M. Scaffolds for engineering novel binding sites in proteins. Curr Opin Struct Biol. 1997;7:463-469.

45. Mathonet P, Fastrez J. Engineering of non-natural receptors. Curr Opin Struct Biol. 2004;14:505-511.

46. $\mathrm{Xu} \mathrm{L}, \mathrm{Aha} \mathrm{P}, \mathrm{Gu} \mathrm{K}$, et al. Directed evolution of high-affinity antibody mimics using mRNA display. Chem Biol. 2002;9:933-942.

47. Högbom M, Eklund M, Nygren PA, Nordlund P. Structural basis for recognition by an in vitro evolved affibody. Proc Natl Acad Sci USA. 2003;100:3191-3196.

48. Tolmachev V, Orlova A, Pehrson R, et al. Radionuclide therapy of HER2-positive microxenografts using a ${ }^{177} \mathrm{Lu}$-labeled HER2-specific Affibody molecule. Cancer Res. 2007;67:2773-2782.

49. Wängler C, Moldenhauer G, Eisenhut M, Haberkorn U, Mier W. Antibodydendrimer conjugates: the number, not the size of the dendrimers, determines the immunoreactivity. Bioconjug Chem. 2008;19:813-820. 